\title{
${ }^{1} \mathrm{H}-\mathrm{NMR}$-based urine metabolomics reveals signs of enhanced carbon and nitrogen recycling in prostate cancer
}

Chiara Bruzzone, Ana Loizaga-Iriarte', Pilar Sánchez-Mosquera, Rubén Gil-Redond, lanire Astobiza, Tammo Diercks, Ana R. Cortazar, Aitziber Ugalde-Olano, Hartmut Schäfer, Francisco J. Blanco, Miguel Unda, Claire Cannet, Manfred Spraul, José M Mato, Nieves Embade, Arkaitz Carracedo* and Oscar Millet*

Table of contents:

Figure S1. DBSCAN cluster analysis.

Figure S2. Cohort/spectrometer comparison.

Figure S3. Multivariate analysis of cancer stages.

Table S1. R2 and Q2 values obtained from multivariate analysis using all the samples from both cohorts.

Table S2. R2 and Q2 values obtained from multivariate analysis using all the samples from both cohorts.

Figure S4. Multivariate analysis of cancer progression indicators using both cohorts. Perineural invasion, Gleason score and PSA were analyzed.

Table S3. R2 and Q2 values obtained from multivariate analysis for different factors of PC aggressiveness.

Figure S5. Supervised multivariate analysis of PC vs BPH patient classified by their BMI using both cohorts independently.

Table S4. Cross validation parameters for comparisons of PC vs BPH patients by their BMI using both cohorts independently.

Figure S6. Multivariate analysis of cancer progression indicators (Gleason score) for both cohorts independently.

Table S5. Cross validation parameters for comparisons between PC and $428 \mathrm{BPH}$ for different9 Gleason scores in both cohorts independently.

Figure S7. Multivariate analysis of PC vs BPH patient classified by the perineural invasion score using both cohorts independently.

Table S6. Cross validation parameters for comparisons between PC and BPH for different perineural invasion scores in both cohorts independently.

Figure S8. Multivariate analysis of cancer progression indicators (PSA levels) using both cohorts independently.

Table S7. R2 and cross validated Q2 and cross-validated accuracies of the PLS comparisons of PC vs BPH patients classified by cancer progression indicators (PSA levels) divided in two different cohorts.

Figure S9. Multivariate analysis of cancer progression indicators. Discriminations inter-groups

Table S8. PLS analysis for discrimination between different factors of PC progression.

Figure S10. Lipid assignment.

Table S9. Univariate analysis using the distinctive peak regions (bins) 
A

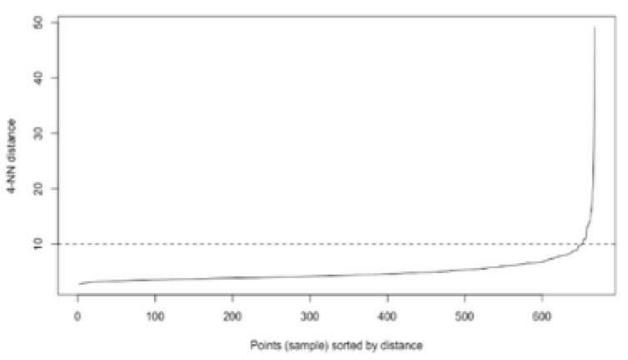

B

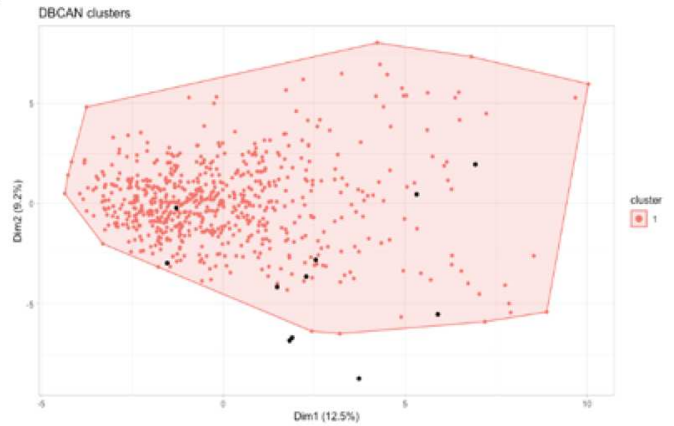

Figure S1. DBSCAN cluster analysis. A. For each sample, distances of the 4 nearest neighbors are represented. Almost are nearest neighbors have a distance lower than 10. B. Principal component analysis $1 \& 2$ for bin intensities, after pareto scaling. DBSCAN identified only 1 cluster of normal samples (red), while those samples that could not be assigned to the main cluster are colored in black. Samples that were considered outliers: AC107, AC192, AC212, AC27, AC282, AC447, AC449, AC452, AC526, AC636, AC652.

A

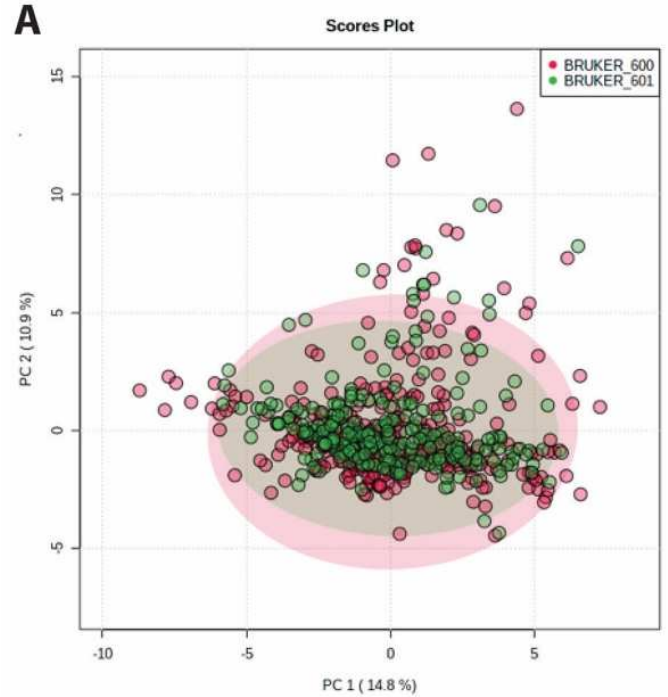

B

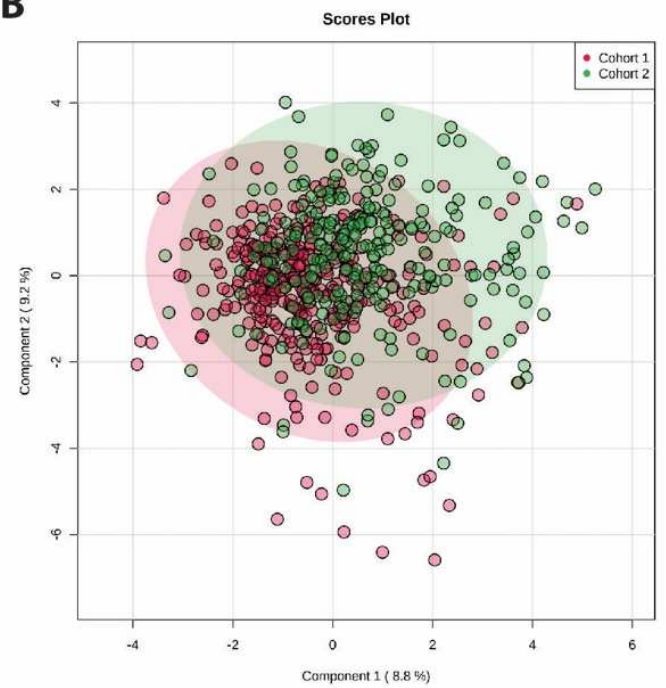

Figure S2. Cohort/spectrometer comparison. PCA (A) and PLS (B) analysis between urine samples of the cohort 1 analyzed in the AVANCE $600 \mathrm{MHz}$ spectrometer (red) and the cohort 2 samples, measured in the IVDr 600 spectrometer (green). 

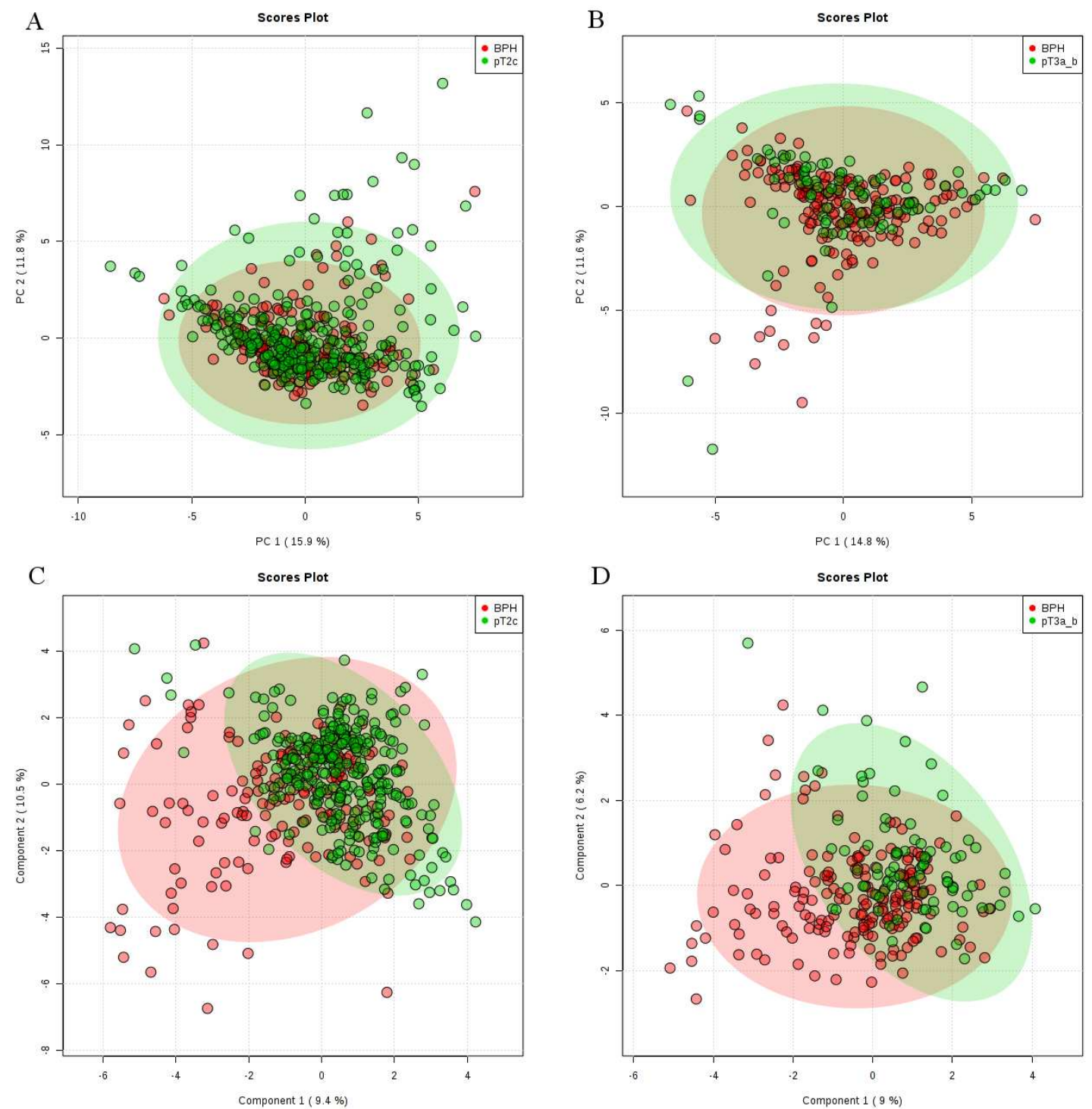

Figure S3. Multivariate analysis of cancer stages. Unsupervised (PCA, A, B) and supervised (PLSDA, C, D) multivariate analysis of the ${ }^{1} \mathrm{H}-\mathrm{NMR}$ bins from urine spectra. A, C. BPH vs prostate cancer patients in $\mathrm{pT} 2 \mathrm{c}$ stage. B, D. BPH vs prostate cancer patients in $\mathrm{pT} 3$ stage.

\begin{tabular}{|l|c|c|c|}
\hline Comparisons & $\mathbf{R}^{\mathbf{2}}$ & $\mathbf{Q}^{\mathbf{2}}$ & Accuracy \\
\hline BPH vs PC patients in pT2c stage & 0.27 & 0.17 & 0.72 \\
\hline BPH vs PC patients in pT3c stage & 0.26 & 0.10 & 0.70 \\
\hline
\end{tabular}

Table S1: $\mathbf{R}^{2}$ and $\mathbf{Q}^{2}$ values obtained from multivariate analysis using all the samples from both cohorts. Both comparisons shown in Figure $\mathrm{S} 3$ did not show a significant discrimination between $\mathrm{BPH}$ and the different PC stages. 


\begin{tabular}{|l|c|c|c|}
\hline Comparisons & $\mathbf{R}^{\mathbf{2}}$ & $\mathbf{Q}^{\mathbf{2}}$ & Accuracy \\
\hline PC vs BPH (lean patients) & 0.36 & 0.16 & 0.77 \\
\hline PC vs BPH (overweight patients) & 0.23 & 0.086 & 0.75 \\
\hline PC vs BPH (obese patients) & 0.156 & -0.04 & 0.65 \\
\hline
\end{tabular}

Table S2: $R^{2}$ and $Q^{2}$ values obtained from multivariate analysis using all the samples from both cohorts. Supervised multivariate analysis (PLS) of the ${ }^{1} \mathrm{H}-\mathrm{NMR}$ bins from urine spectra of PC vs BPH patient subcohorts, classified by their BMI.
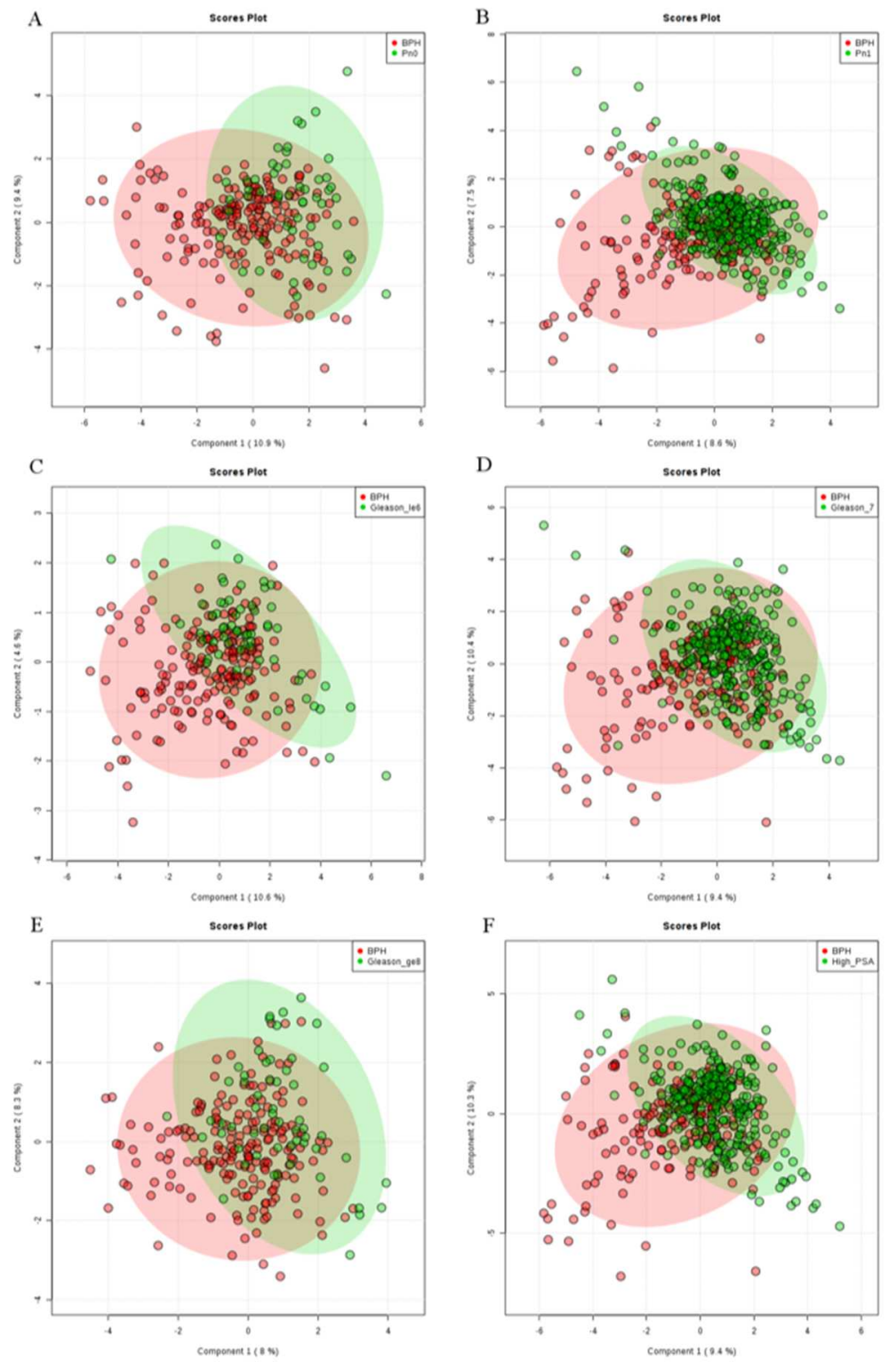

Figure S4. Multivariate analysis of cancer progression indicators using both cohorts. Supervised (PLS-DA) multivariate analysis of the ${ }^{1} \mathrm{H}-\mathrm{NMR}$ bins from urine spectra for $\mathrm{BPH}$ (always in red) vs perineural invasion (A, B), Gleason score $(\mathbf{C}, \mathbf{D}, \mathbf{E})$ and PSA (F), as indicated in the legends. (Gleason_le6: Gleason score lower or equal to 6; Gleason_7: Gleason score equal to 7; Gleason_ge8: Gleason score greater or equal to 8; High PSA: more than $10 \mathrm{ng} / \mathrm{mL}$ ). 


\begin{tabular}{|l|c|c|c|}
\hline Comparisons & $\mathbf{R}^{\mathbf{2}}$ & $\mathbf{Q}^{\mathbf{2}}$ & Accuracy \\
\hline BPH vs perineural invasion (pn0) & 0.17 & 0.10 & 0.77 \\
\hline BPH vs perineural invasion (pn1) & 0.27 & 0.19 & 0.73 \\
\hline BPH vs Gleason score (level 6) & 0.12 & 0.02 & 0.77 \\
\hline BPH vs Gleason score (level 7) & 0.03 & 0.19 & 0.70 \\
\hline BPH vs Gleason score (level 8) & 0.11 & -0.10 & 0.77 \\
\hline BPH vs High PSA & 0.27 & 0.18 & 0.71 \\
\hline
\end{tabular}

Table S3: $R^{2}$ and $Q^{2}$ values obtained from multivariate analysis for different factors of $P C$ aggressiveness. Supervised multivariate analysis (PLS) of the ${ }^{1} \mathrm{H}-\mathrm{NMR}$ bins from urine spectra of PC vs BPH patient subcohorts, classified according to the aggressiveness factors: perineural invasion, Gleason score and PSA.
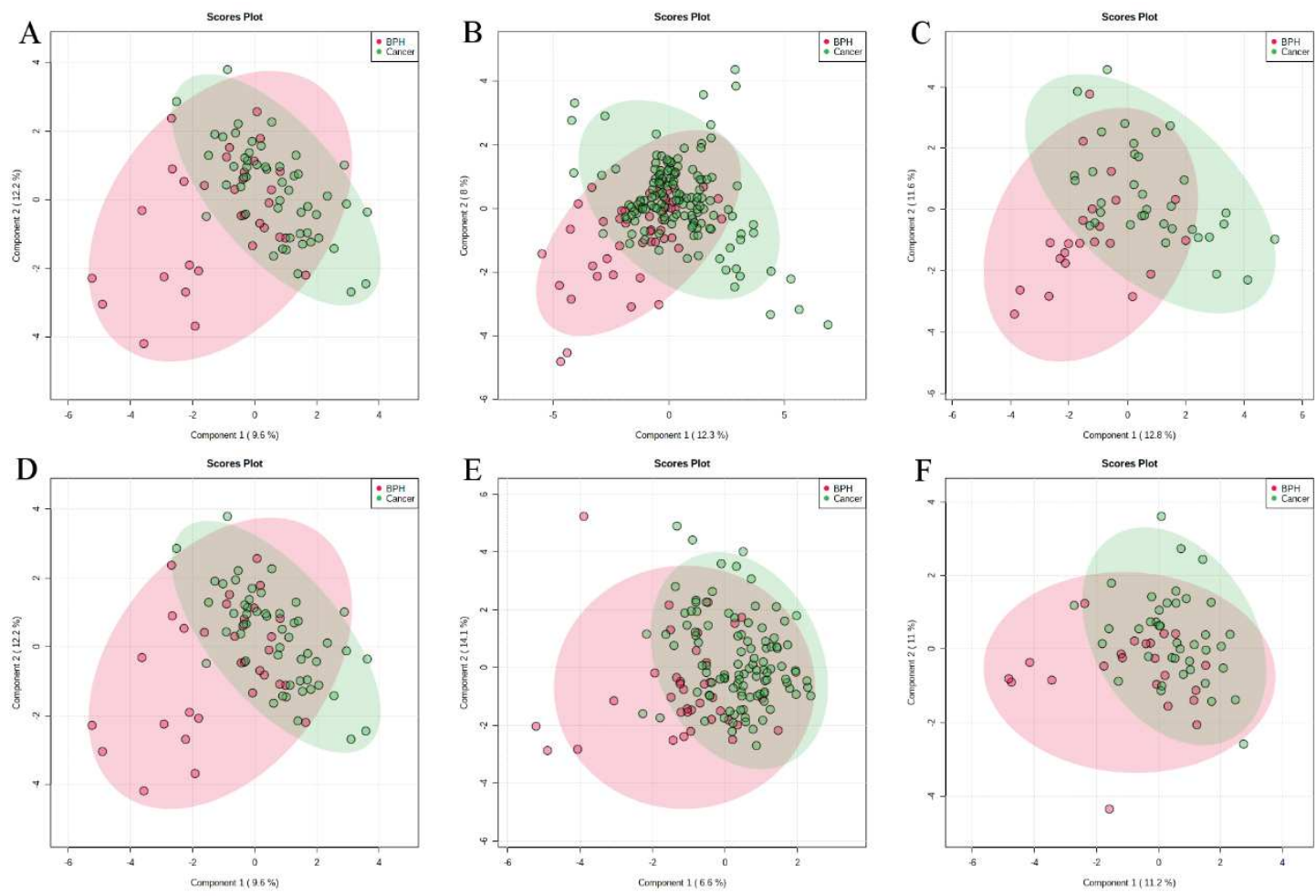

Figure S5. Supervised multivariate analysis of PC vs BPH patient classified by their BMI using both cohorts independently. Supervised multivariate analysis (PLS) of the ${ }^{1} \mathrm{H}-\mathrm{NMR}$ bins from urine spectra of PC vs BPH patients from cohort 1 (A-C) and cohort 2 (D-E) independently, classified by their BMI. PLS-DA obtained after comparison of lean (BMI $<25$, A cohort 1, D cohort 2 ), overweight ( $25<\mathrm{BMI}<30, \mathrm{~B}$ cohort $1, \mathbf{E}$ cohort 2$)$ or obese (BMI $>30, \mathrm{C}$ cohort $1, \mathbf{F}$ cohort 2 ) individuals suffering from $B P H$ and $P C$. 


\begin{tabular}{|l|c|c|c|}
\hline Comparisons & $\mathbf{R}^{\mathbf{2}}$ & $\mathbf{Q}^{\mathbf{2}}$ & Accuracy \\
\hline Cohort 1 - PC vs BPH (lean) & 0.46 & 0.20 & 0.81 \\
\hline Cohort 1 - PC vs BPH (overweight) & 0.27 & 0.07 & 0.78 \\
\hline Cohort 1 - PC vs BPH (obese) & 0.25 & -0.13 & 0.61 \\
\hline Cohort 2 - PC vs BPH (lean) & 0.31 & 0.01 & 0.65 \\
\hline Cohort 2 - PC vs BPH (overweight) & 0.32 & 0.10 & 0.75 \\
\hline Cohort 2 - PC vs BPH (obese) & 0.17 & -0.20 & 0.6 \\
\hline
\end{tabular}

Table S4. Cross validation parameters for comparisons of PC vs BPH patients by their BMI using both cohorts independently. Supervised multivariate analysis (PLS) of the ${ }^{1} \mathrm{H}-\mathrm{NMR}$ bins from urine spectra of PC vs BPH patient subcohorts, classified according to the BMI: perineural lean, overweight and obese.
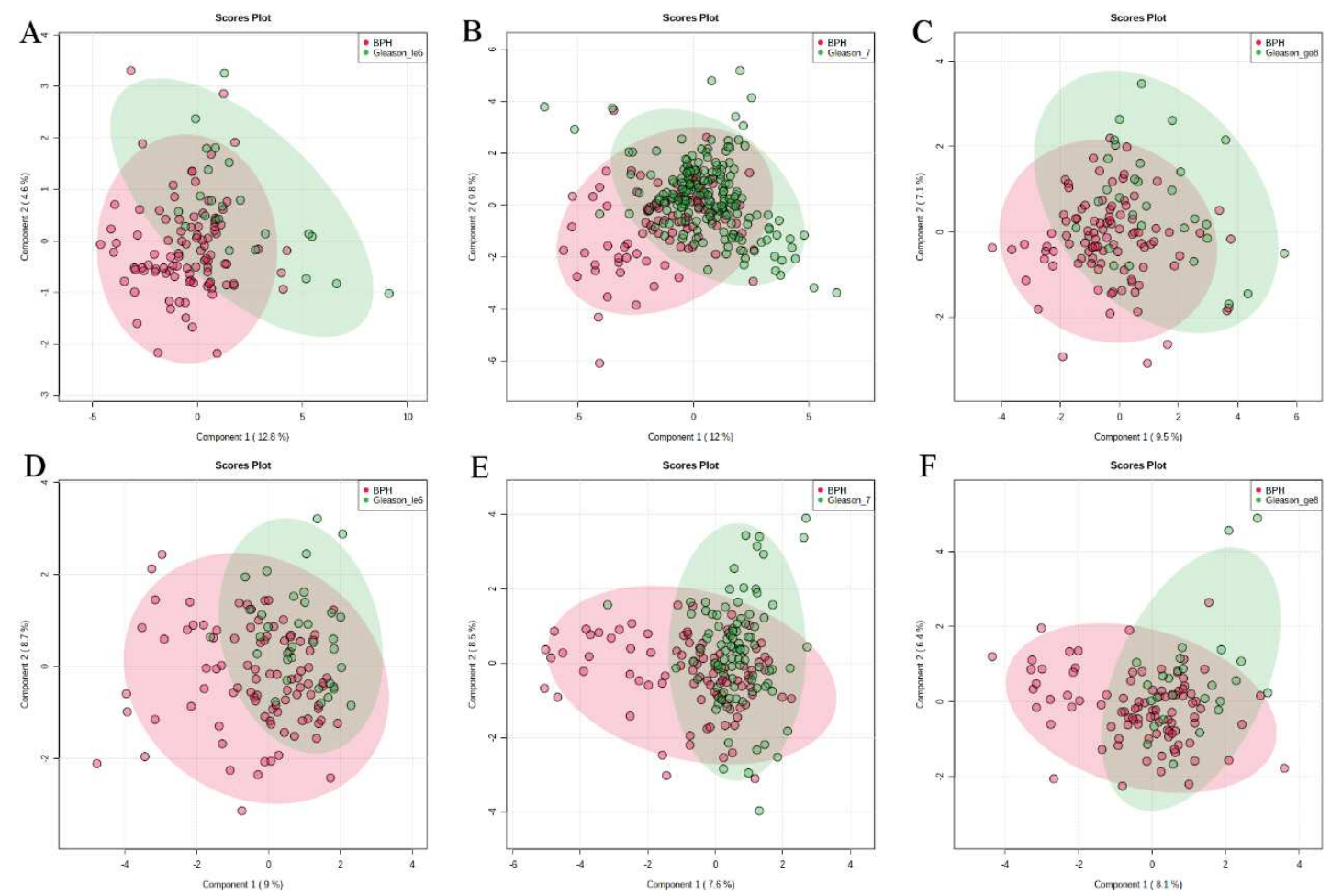

Figure S6. Multivariate analysis of cancer progression indicators (Gleason score) for both cohorts independently. Supervised (PLS-DA) multivariate analysis of the ${ }^{1} \mathrm{H}-\mathrm{NMR}$ bins from urine spectra for BPH (always in red) vs Gleason score (A, B, C Cohort 1 and D, E, F Cohort 2) as indicated in the legends. (Gleason_le6: Gleason score lower or equal to 6; Gleason_7: Gleason score equal to 7; Gleason_ge8: Gleason score greater or equal to 8) 


\begin{tabular}{|l|c|c|c|}
\hline Comparisons & $\mathbf{R}^{\mathbf{2}}$ & $\mathbf{Q}^{\mathbf{2}}$ & Accuracy \\
\hline Cohort 1 - BPH vs Gleason_le6 & 0.22 & 0.12 & 0.81 \\
\hline Cohort 1 - BPH vs Gleason_7 & 0.34 & 0.18 & 0.74 \\
\hline Cohort 1 - BPH vs Gleason_ge8 & 0.17 & -0.11 & 0.74 \\
\hline Cohort 2 - BPH vs Gleason_le6 & 0.14 & -0.16 & 0.70 \\
\hline Cohort 2 - BPH vs Gleason_7 & 0.26 & 0.09 & 0.63 \\
\hline Cohort 2 - BPH vs Gleason_ge8 & 0.14 & -0.16 & 0.74 \\
\hline
\end{tabular}

Table S5. Cross validation parameters for comparisons between PC and BPH for different Gleason scores in both cohorts independently. Supervised multivariate analysis (PLS) of the ${ }^{1} \mathrm{H}$ NMR bins from urine spectra of PC vs BPH patient subcohorts, classified according to the aggressiveness factor: Gleason score.
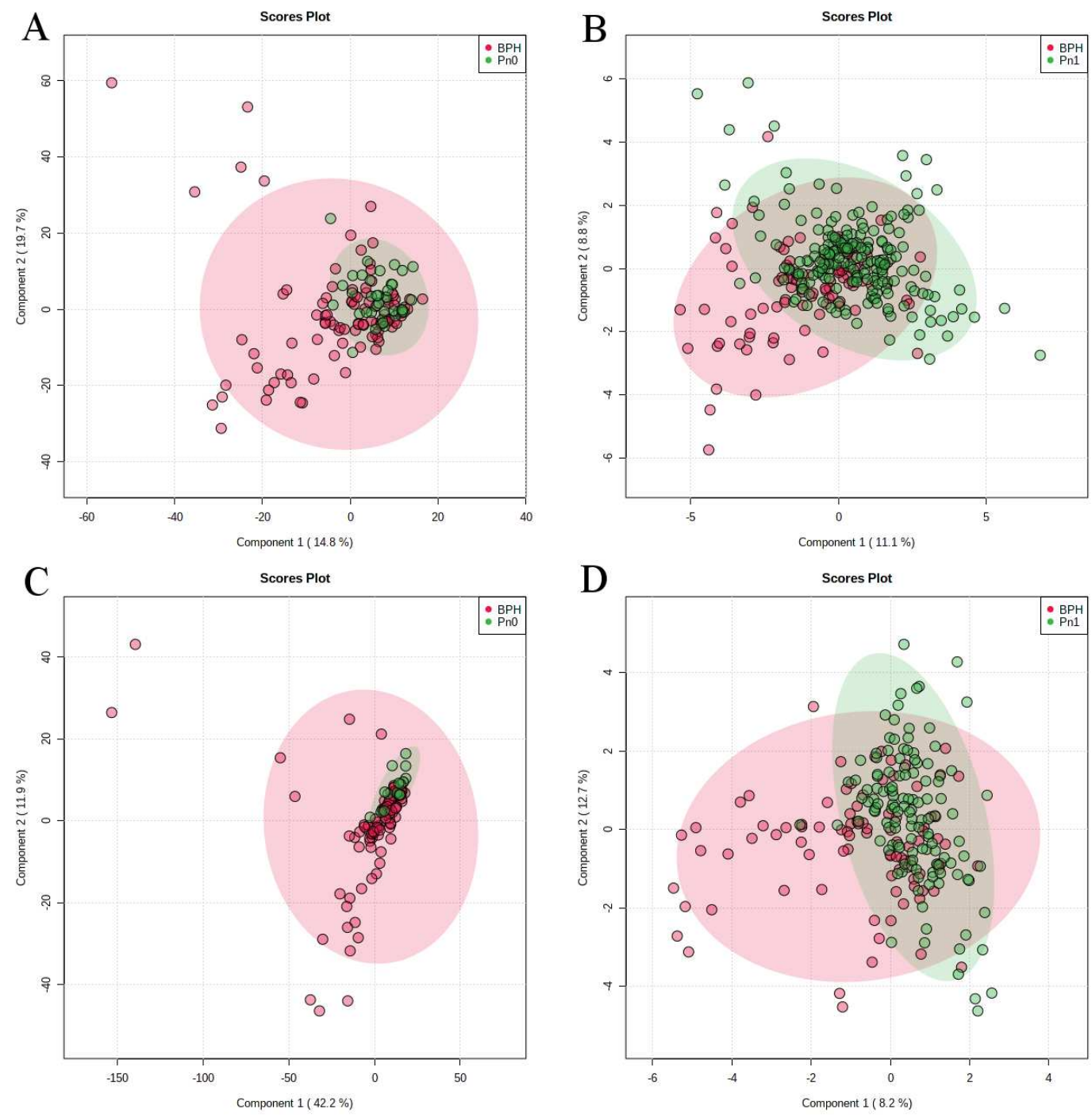

Figure S7. Multivariate analysis of PC vs BPH patient classified by the perineural invasion score using both cohorts independently. Supervised (PLS-DA) multivariate analysis of the ${ }^{1} \mathrm{H}-\mathrm{NMR}$ bins from urine spectra for BPH (always in red) vs perineural invasion (A, B, Cohort $1 ; C, D$, Cohort 2). 


\begin{tabular}{|l|c|c|c|}
\hline Comparisons & R2 & Q2 & Accuracy \\
\hline Cohort 1 - BPH vs Pn0 & 0.12 & 0.04 & 0.72 \\
\hline Cohort 1 - BPH vs Pn1 & 0.35 & 0.17 & 0.71 \\
\hline Cohort 2 - BPH vs Pn0 & 0.04 & -0.02 & 0.86 \\
\hline Cohort 2 - BPH vs Pn1 & 0.25 & 0.12 & 0.70 \\
\hline
\end{tabular}

Table S6. Cross validation parameters for comparisons between PC and BPH for different perineural invasion scores in both cohorts independently. Supervised multivariate analysis (PLS) of the ${ }^{1} \mathrm{H}-\mathrm{NMR}$ bins from urine spectra of PC vs BPH patient subcohorts, classified according to the aggressiveness factor: perineural factor.
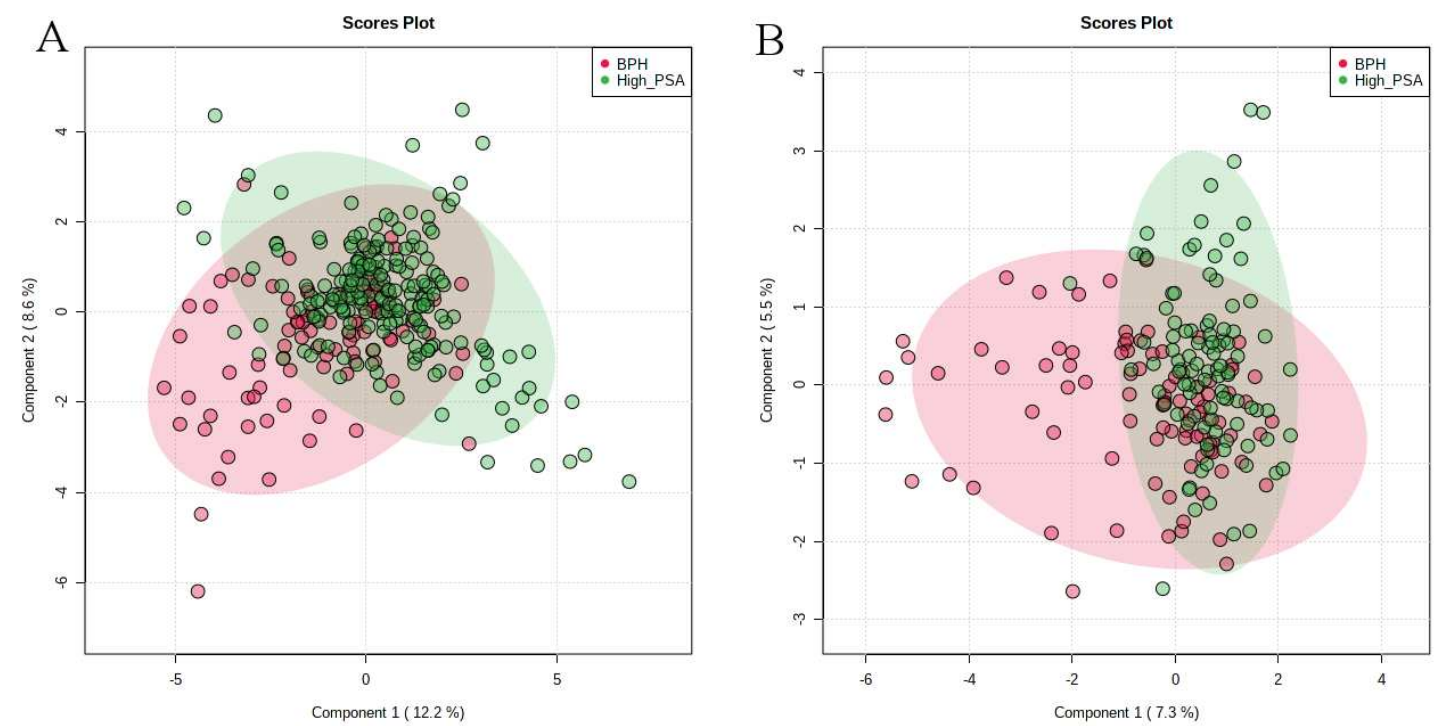

Figure S8. Multivariate analysis of cancer progression indicators (PSA levels) using both cohorts independently. Supervised (PLS-DA) multivariate analysis of the ${ }^{1} \mathrm{H}-\mathrm{NMR}$ bins from urine spectra for BPH vs PSA levels (A,Cohort 1 and B, Cohort 2) as indicated in the legends (High PSA: more than $10 \mathrm{ng} / \mathrm{mL}$ ).

\begin{tabular}{|l|c|c|c|}
\hline Comparisons & R2 & Q2 & Accuracy \\
\hline Cohort 1 - BPH vs High PSA & 0.36 & 0.15 & 0.71 \\
\hline Cohort 2 - BPH vs High PSA & 0.28 & 0.08 & 0.66 \\
\hline
\end{tabular}

Table S7. R2 and cross validated Q2 and cross-validated accuracies of the PLS comparisons of PC vs BPH patients classified by cancer progression indicators (PSA levels) divided in two different Cohorts. 

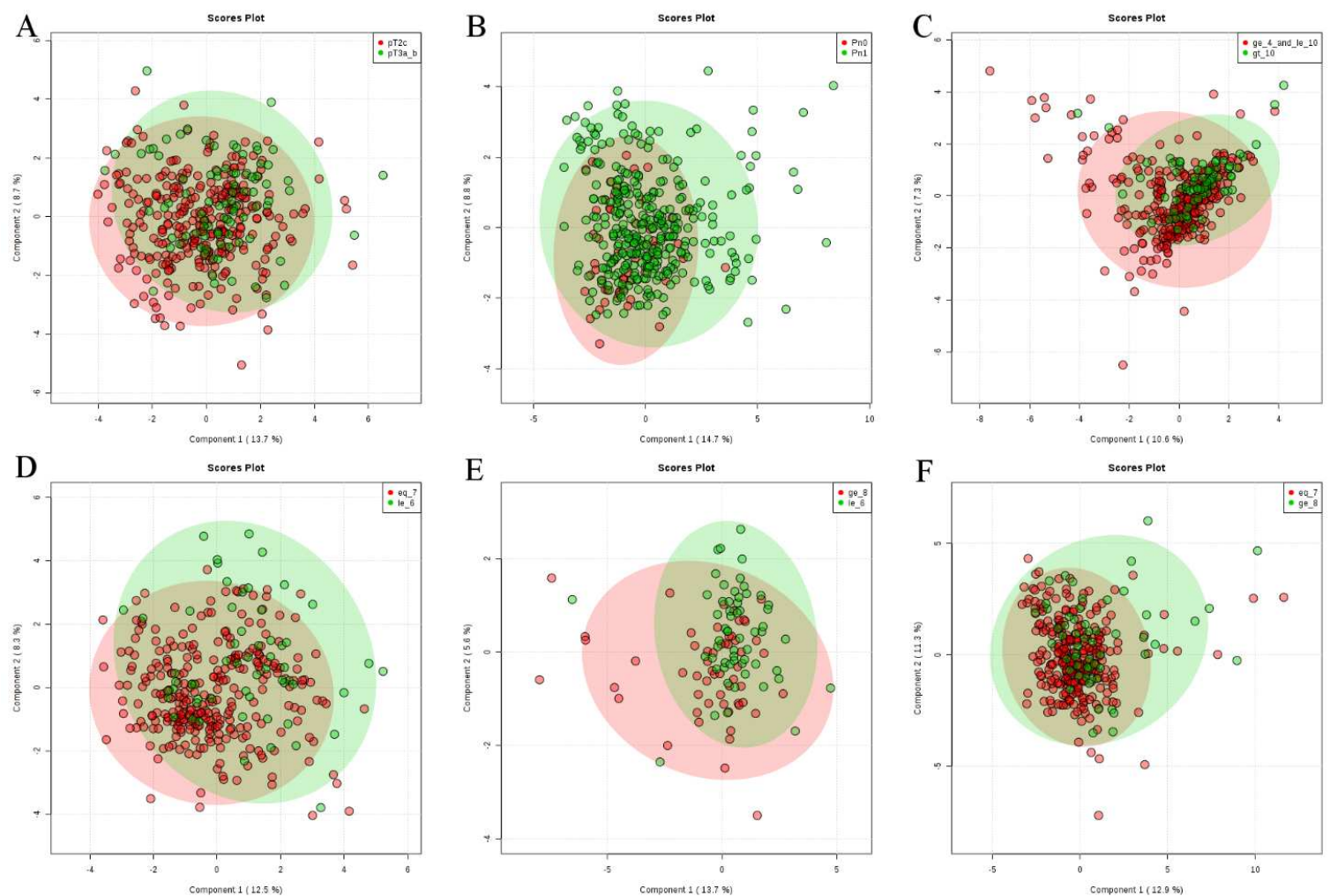

Figure S9. Multivariate analysis of cancer progression indicators. Discriminations inter-groups.

Supervised (PLS-DA) multivariate analysis of the ${ }^{1} \mathrm{H}-\mathrm{NMR}$ bins from urine spectra for cancer stage pT2c vs pT3a_b (A); perineural invasion (B), High PSA vs Very high PSA (C) Gleason_le6 vs Gleason_7 (D); Gleason_ge8 vs Gleason_le6 (E), Gleason_ge8 vs Gleason_7 (F) as indicated in the legends. (Gleason_le6: Gleason score lower or equal to 6; Gleason_7: Gleason score equal to 7; Gleason_ge8: Gleason score greater or equal to 8; High PSA: more than $10 \mathrm{ng} / \mathrm{mL}$ ).

\begin{tabular}{|l|c|c|c|}
\hline Comparisons & R2 & Q2 & Accuracy \\
\hline pT2c vs pT3a_b cancer stage & 0.03 & -0.04 & 0.77 \\
\hline Pn 0 vs Pn 1 & 0.03 & -0.01 & 0.86 \\
\hline Lower PSA vs High PSA levels & 0.05 & -0.03 & 0.81 \\
\hline Gleason_le6 vs Gleason_7 & 0.13 & -0.03 & 0.83 \\
\hline Gleason_ge8 vs Gleason_le6 & 0.10 & -0.08 & 0.44 \\
\hline Gleason_ge8 vs Gleason_7 & 0.06 & -0.00 & 0.84 \\
\hline
\end{tabular}

Table S8. PLS analysis for discrimination between different factors of PC progression. R2 and cross validated Q2 and cross-validated accuracies of the PLS comparisons inter-groups of patients classified by cancer stages and progression indicators (perineural invasion, PSA level, Gleason score). 


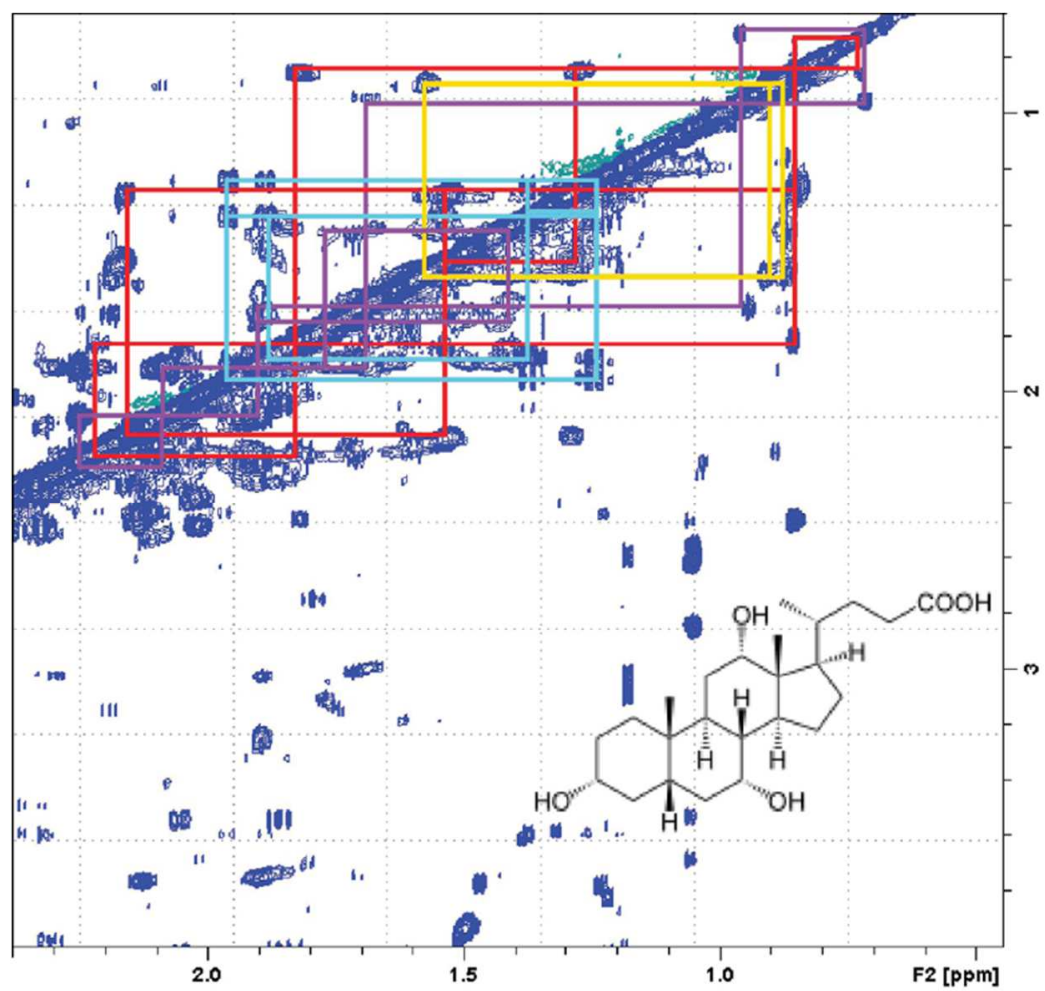

Figure S10 Lipid assignment. Expansion of a TOCSY spectrum of a urine sample connecting crosspeaks that are compatible with the steroid scaffold found in cholesterol and derivatives. Colors of the lines reflect the different spin system of the molecule. 


\begin{tabular}{|l|l|l|}
\hline Bins & Fold change & p-value \\
\hline bin_00.53 & -1.329 & $1.08 \mathrm{E}-09$ \\
\hline bin_00.56 & -0.810 & $1.43 \mathrm{E}-05$ \\
\hline bin_00.59 & -0.979 & $7.27 \mathrm{E}-06$ \\
\hline bin_00.62 & -0.997 & $1.67 \mathrm{E}-09$ \\
\hline bin_00.65 & -0.767 & $1.67 \mathrm{E}-09$ \\
\hline bin_00.68 & -0.731 & $2.41 \mathrm{E}-08$ \\
\hline bin_00.71 & -0.632 & $7.61 \mathrm{E}-09$ \\
\hline bin_00.74 & -0.522 & $1.95 \mathrm{E}-06$ \\
\hline bin_01.94 & -0.545 & $2.53 \mathrm{E}-09$ \\
\hline bin_02.90 & -0.361 & $3.36 \mathrm{E}-02$ \\
\hline bin_03.08 & 0.357 & $3.40 \mathrm{E}-02$ \\
\hline bin_06.98 & -0.315 & $6.81 \mathrm{E}-04$ \\
\hline bin_07.16 & -0.328 & $9.67 \mathrm{E}-03$ \\
\hline bin_07.46 & -0.314 & $4.04 \mathrm{E}-06$ \\
\hline bin_07.49 & -0.552 & $2.29 \mathrm{E}-06$ \\
\hline bin_07.52 & -0.614 & $1.85 \mathrm{E}-08$ \\
\hline bin_07.61 & -0.459 & $1.28 \mathrm{E}-05$ \\
\hline bin_07.88 & -0.601 & $1.57 \mathrm{E}-03$ \\
\hline bin_07.91 & -0.827 & $4.23 \mathrm{E}-08$ \\
\hline bin_08.18 & -0.303 & $4.64 \mathrm{E}-02$ \\
\hline bin_08.27 & -0.339 & $8.45 \mathrm{E}-03$ \\
\hline bin_08.60 & -0.396 & $1.23 \mathrm{E}-05$ \\
\hline bin_08.63 & -0.512 & $5.55 \mathrm{E}-07$ \\
\hline bin_08.66 & -0.450 & $1.48 \mathrm{E}-04$ \\
\hline bin_08.69 & -0.612 & $7.39 \mathrm{E}-06$ \\
\hline bin_08.72 & -0.516 & $9.45 \mathrm{E}-06$ \\
\hline bin_08.75 & -0.750 & $4.22 \mathrm{E}-07$ \\
\hline bin_08.78 & -0.851 & $4.00 \mathrm{E}-08$ \\
\hline bin_08.81 & -0.370 & $1.59 \mathrm{E}-02$ \\
\hline bin_08.96 & -0.746 & $2.39 \mathrm{E}-07$ \\
\hline bin_09.02 & -0.512 & $1.94 \mathrm{E}-04$ \\
\hline bin_09.05 & -0.348 & $3.44 \mathrm{E}-03$ \\
\hline bin_09.08 & -0.879 & $6.27 \mathrm{E}-05$ \\
\hline bin_09.11 & -0.664 & $3.09 \mathrm{E}-05$ \\
\hline bin_09.17 & -0.958 & $8.46 \mathrm{E}-06$ \\
\hline bin_09.20 & -0.963 & $4.38 \mathrm{E}-07$ \\
\hline bin_09.23 & -0.884 & $1.36 \mathrm{E}-05$ \\
\hline bin_09.26 & -0.784 & $6.02 \mathrm{E}-05$ \\
\hline bin_09.32 & -0.725 & $4.77 \mathrm{E}-05$ \\
\hline bin_09.35 & -0.748 & $4.08 \mathrm{E}-05$ \\
\hline bin_09.38 & -0.572 & $5.93 \mathrm{E}-07$ \\
\hline bin_09.41 & -0.765 & $1.27 \mathrm{E}-05$ \\
\hline bin_09.44 & -0.641 & $5.25 \mathrm{E}-04$ \\
\hline bin_09.47 & -0.837 & $1.27 \mathrm{E}-06$ \\
\hline & & \\
\hline
\end{tabular}

Table S9: Univariate analysis using the distinctive peak regions (bins). Wilcoxon Mann Whitney test classified a set of bins as statistically significant, with acceptable false discovery rates ( $p$ value $<0.05$ ) and $\log 2 \mathrm{FC}$ above 0.3 . 